5. STELLAR EVOLUTION 


\title{
THE CHEMICAL EVOLUTION OF THE MAGELLANIC CLOUDS
}

\author{
F. SPITE, M. SPITE \\ Observatoire de Paris \\ 92195 Meudon Cedex \\ France
}

\begin{abstract}
New observations give more details and some confirmation about the chemical evolution of the Clouds. From more numerous data, information is beginning to emerge about the importance of mixing in the Clouds.
\end{abstract}

\section{Introduction}

The progressive enrichment of the Galaxy in heavy elements, built from hydrogen, has been the subject of a number of studies. Well known are the works of Audouze \& Tinsley (1976) and of many others. A number of the basic parameters of the models are uncertain. It is therefore particularly interesting to check how the Galactic models of chemical evolution can be adapted to the Magellanic Clouds, even if the comparison implies the difficult observation of remote and faint objects. The Clouds have both advantages and disadvantages.

The ideal data to collect for a comparison with theory would be a list of the abundances as a function of position and of time. In our Galaxy, the radial gradients of abundance have been used as an additional constraint in the models by Matteucci \& Francois (1989). In the Clouds, no such symmetry is present. Fortunately, also in our Galaxy, the kinematical (and dynamical) parameters of the field stars are linked statistically with their age. Our present knowledge of the complicated kinematical structure of the Clouds means that no such statistical connection can be used as a guide.

But the Clouds have an advantage: their large distance (large relative to the "depth" of the Clouds) means that, with some care, the distances of individual stars within each Cloud may be taken as equal. The colour-magnitude diagram of the field stars is then similar to the colourmagnitude of a cluster, and with some care, some of its parameters (age, metallicity) can be estimated. The possibility of knowing the luminosity of the observed objects is important: for example, a) Azzopardi et al. (1988) stressed an important problem about the evolution of the WR stars which, in turn, have an important role in the synthesis of elements, and b) Smith \& Lambert (1989) measured the lithium abundance in a sample of AGB stars of the Small Magellanic Cloud (SMC) in a well-defined phase of their evolution .

The subject of the chemical evolution is too large to be covered in a few pages. We will therefore rely heavily on the papers of Feast (1989) and Westerlund (1990), concentrating on recent works. The attentive reader will notice much borrowing from the papers presented in the colloquium (hereafter "Paris Colloquium") held in Paris (de Boer et al. 1989 and this paper will have to be complemented by the many new results presented in the symposium. 


\section{The data}

\subsection{THE CLUSTERS}

An important part of the data comes from the Clusters, for which both age and metallicity can be estimated simultaneously. Lists of clusters (with their main parameters such as age and metallicities) were published recently by Westerlund and Feast (as mentioned above), and also by Sagar \& Pandey (1989) and by Seggewiss \& Richtler (1989). More data were presented at this symposium (e.g. Da Costa, and Schommer et al.). Most clusters seem to follow an age-metallicity relation.

\subsection{OLD POPULATIONS}

The clusters are important tracers of chemical evolution. However, the bulk of the processed matter is locked up in the field stars. Normal RR Lyrae stars are examples of the old metal-poor populations. They seem to indicate ages and metallicities similar to those found in the old halo population of our Galaxy. Butler et al. (1982) used the $\Delta S$ method for the RR Lyrae stars to determine abundance, and gauged the age from the colours and periods of the variables. The determinations are based on calibrations made in our Galaxy, which should apply to Magellanic objects since they are very similar: the metallicity is found to be about -1.4 dex. For example, old field stars have been traced by:

- Hardy et al. (1984) in the Large Magellanic Cloud (LMC) Bar (they found stars older than $3 \times 10^{9}$ years in very small proportion).

- Rich et al. (1984) in the SMC near the cluster Kron 3. Here the proprotion is non-negligible; the authors find that the age is larger than the age of the cluster, i.e. larger than $10^{9}$ years. The metallicity of this population is lower than the metallicity $([\mathrm{Fe} / \mathrm{H}]=-1.2 \mathrm{dex}$ ) of the cluster (we use here the classical notation $[\mathrm{X}]=\log \mathrm{X}_{*-\log \mathrm{X}_{\mathrm{o}}}$ ).

- Stryker et al. (1985) in the SMC, near the cluster NGC 121. The metallicity is found from comparison with the cluster and should be near $[\mathrm{Fe} / \mathrm{H}]=-1.7$ dex. The proportion of old stars is rather important. The age is found by comparison with the cluster and could be $12 \times 10^{9}$ years.

- Bolte (1987) found in the halo of the SMC a small proportion of young stars (age $<0.3 \times 10^{9}$ years) of metallicity -0.6 dex. The larger proportion is made up of stars which could be as deficient as -1.3 dex and with an age of between 8 and $14 \times 10^{9}$ years.

More recent work was presented at the "Paris Colloquium" and at this symposium.

In conclusion, in the SMC the old population dominates, but in the LMC the intermediate age population dominates.

\subsection{INTERMEDIATE AGE OBJECTS}

In all surveys of field stars, a conspicuous population of stars of intermediate age is found. It is difficult to assign definite ages and metallicities to these continuous distributions. Hardy et al. (1984) found that the population of the bar of the LMC is essentially a population younger than the solar neighbourhood $\left(10^{9}<\right.$ age $<3 \times 10^{9}$ years) with a metallicity assumed to be -0.7 dex.

There is a large range of metallicity in the intermediate age stars and it is not possible to derive any valuable information about the degree of mixing (homogenization of the abundances).

Most of the red giants of the Clouds are of intermediate age. Red variables were observed and found to be in two age groups.

The bulk of the Planetary Nebulae have ages around 3.5 Gyr. The precursors should be stars of intermediate age population (Meatheringham et al. 1988). A considerable body of observations 
has been obtained recently by several astronomers; the abundances of elements, other than $\mathrm{C}, \mathrm{N}$ and $\mathrm{O}$, give some information on the abundances of their precursors.

\subsection{YOUNG OBJECTS}

HII regions. The abundances in these young objects have been reviewed by Dufour (1984) and Dennefeld (1989), who included in the discussion Mathis' (1985) results. Little has been published since. The link built by Russell and Dopita (1990a, b) between the abundances in HII regions, SN remnants, $\mathrm{H}-\mathrm{H}$ objects and stars is a very useful tool, and is discussed in this symposium. One of the advantages of the HII regions is that they are observable even in external galaxies.

Young clusters. The work of Cohen (1982) shows that the young clusters in the LMC have a metallicity of about -0.4 dex.

Variable stars. The study of Cepheids indicates a metallicity of about -0.2 dex for the LMC, and -0.6 for the SMC (Feast 1989).

Non-variable stars. Technical progress has enabled the spectra of some of the bright stars to be obtained. It is recommended that the very brightest ones be avoided, as these could have unstable atmospheres. Modern observations concern cool supergiants with atmospheres rather similar to the atmospheres of the classical luminous giants of the Galactic globular clusters (Russell \& Bessell 1989, Spite et al. 1989a, b).

Abundance analyses in supergiants are not easy. It is well known that the termperature calibration of the supergiant colours is not well defined. The reddening of individual stars in the Clouds is also not well defined. However, the quality of the spectroscopic observations is nowadays good enough to provide a check on the effective temperature of the model, using the excitation temperature. Note that the abundances are determined differentially, relative to the abundance of a galactic supergiant (Canopus). Finally, the ratio of the abundances of two elements is more stable than the abundances themselves, at least when the individual abundances are measured from lines of similar atomic properties (similar levels, similar ionization potential, etc.).

The results of these abundance determinations are that the metallicity of the young field stars is about -0.3 dex in the LMC and -0.65 dex in the SMC (Russell \& Bessell 1989, Spite et al. 1989). The scatter is moderate: $\sigma=0.2 \mathrm{dex}$. We will come back to this point.

For elemental abundances, the main feature is a normal stellar abundance of $\mathrm{C}$, in contrast with the large anomaly noted in the HII regions. For other elements, the stellar abundances agree with those in the HII regions (Fig. 1). Also, there is smaller deficiency of the heavy elements, and the light metals, in the metal-poor stars of our Galaxy, show a deficiency smaller than the $\mathrm{Fe}$ deficiency. Russell \& Dopita (1990a) remark that this feature is not seen in the differential comparison with Canopus. This fact may have several interpretations.

These various analyses (different spectrographs, different authors, atomic and molecular lines) show an excellent coherence, and give confidence in the results. In particular, they give confidence in the result about $\mathrm{C}$, even if the stellar value differs from the HII regions value. Another argument about the reliability of the normal $\mathrm{C}$ abundance is derived from the stellar $\mathrm{S}$ abundance (Spite \& Spite, 1990).

Interstellar medium. Observations of the ISM in the optical domain, in the UV, in the IR, in radio (millimetric, centimetric) have considerably increased in the last years. The synthesis of the derived abundances of the elements is essentially in agreement with the abundances obtained from the young field stars. 


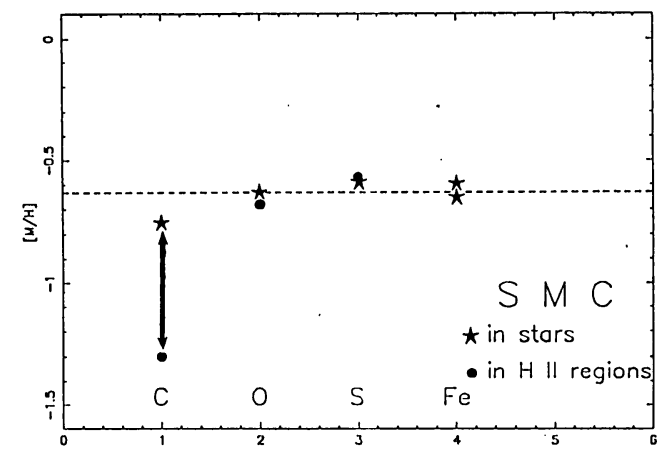

Figure 1. Deficiences $[M / H]$ of the elements $C, O$ and $S$ in the SMC, measured in field stars and in HII regions. Stellar deficiences of Fe by Spite $e t$ al. and by Russell \& Bessell are shown for comparison. No $\mathrm{C}$ anomaly is found in the stars.

\section{The case of the young blue globular clusters}

Photometry (Richtler \& Nelles 1983) and spectroscopic observations in two blue globular clusters, NGC 330 (SMC) and NGC 1818 (LMC) have shown that the observed stars were metal-poor, more metal deficient than the field stars of about the same age.

The determination of the reddening is a difficult problem. For example, NGC 330 is embedded in gas (Bica et al. 1990). However, taking into account two possible values of the reddening, the resulting abundances are not very different, and the relative abundances (ratios) are reasonably stable.

Observations have been published (Jüttner et al. 1989, Reitermann et al. 1990) for one hot star in each of the two young blue globular clusters. The authors find a metallicity around $-0.8 \mathrm{dex}$ for NGC 1818 and -1.1 dex for NGC 330 (see paper in this symposium), but C is more deficient and $\mathrm{N}$ less deficient. One possible explanation could be some mixing with the products of the CNO cycle.

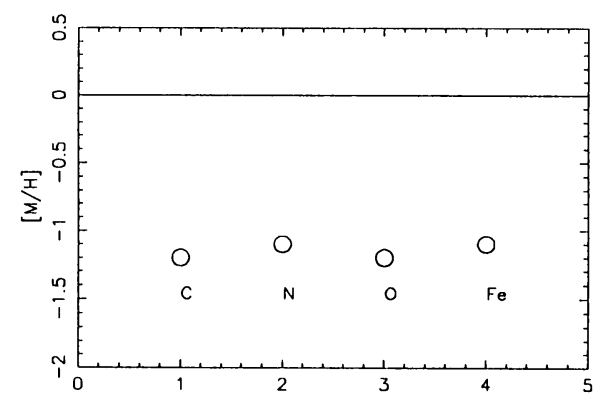

Figure 2. Deficiences $[M / H]$ of the elements $C, N, O$ and $\mathrm{Fe}$ in the star NGC 330:A7. Good agreement is found between atomic and molecular abundances of $\mathrm{C}$ and $\mathrm{N}$. The relative abundances are nearly solar. 
Observations have been published (Spite et al. 1986, Richtler et al. 1989) for one cool star in each of these clusters. Additional observations have been made in the frame of the ESO key-program. The low metallicity of the cluster NGC 330 is confirmed (around -1.0 dex) for both cool and hot stars. Two intermediate temperature stars have also been observed. The definitive individual abundances of the elements are under study (Richtler et al. 1990, Wolf et al. 1990).

The $\mathrm{C}$ deficiency in the cool stars is about the same as the Fe deficiency. This is confirmed by the analysis of molecular bands in the star NGC 330:A7 (Barbuy et al. 1990), who also find nearly the same deficiency for $\mathrm{N}$. The agreement between the molecular and atomic abundances of $\mathrm{C}$ may be regarded as a proof of the reliability of the abundance determination in such stars, giving confidence in the results, especially in the nearly solar relative abundances of $\mathrm{C}, \mathrm{N}, \mathrm{O}$ and $\mathrm{Fe}$ (Fig. 2).

The results of the spectroscopic analysis of the two blue globular clusters observed tend to show that the matter of the two clusters has not been mixed to the matter of the Clouds. The MCs are not mixed by the powerful differential rotation which stirs the objects in our Galaxy. The Clouds are less massive and exert smaller tidal forces. It is, therefore, not so surprising to see a relatively nearby cluster isolated from the main galaxy. Note, however, that adopting a large reddening, Bessell (1990) finds for the stars of NGC 330 a metallicity similar to the metallicity of the young field stars. The large value of the reddening is found by a comparison of Magellanic stars with Galactic stars, which is open to discussion. This point deserves further study.

\section{The evolution of the metallicity of the Clouds}

Feast (1989) proposed for the mean metal-enrichment curve, two logarithmic curves, which follow the general trend quite well. It is interesting to compare these curves with the models of chemical evolution computed by Russell \& Dopita (1990b) for the Clouds, and for the Galaxy. In both cases, NGC 330 is far from the mean curve, and considered a "discrepancy". It is the younger of the metal-poor clusters in the evolution diagram of the SMC, (but not the only one). The situation is the same in the diagrams of other authors (e.g. Stryker et al. 1985, Butler et al. 1982). It suggests the following scenario. Rather than admit a quite low mean metallicity, suddently increasing to reach the metallicity of the young objects, it would be preferable to assume two lines: one, nearly horizontal for the metal-poor clusters of various ages, and one increasing progressively, corresponding to the well-mixed regions. This would imply that some regions of the Clouds are not well mixed. Admittedly the rather close values (as noted above) of the metallicities of the field supergiants show the existence of a rather efficient mixing; also Pagel $e t$ al. (1978) noted the homogeneity of the abundances of the HII regions (no gradient). But if some mixing is obviously present in most parts of the Clouds, it does not necessarily take place everywhere. Some theories of galaxy formation rely on the progressive accretion of unprocessed material or of only slightly processed material (Cayrel 1986). Also, in our Galaxy, the peculiarities of the cluster Pal. 12 have been interpreted by a low metallicity and a shorter age: shorter than the mean age of the clusters of the same (low) metallicity (Gratton \& Ortolani 1988).

In view of the pending problem, the observation of other stars in the field and in the clusters would be extremely important.

\section{References}

Audouze, J., Tinsley, B. (1976), Ann. Rev. Astron. Astrophys. 14, 43. Azzopardi, M., Lequeux, J., Maeder, A. (1988), Astron. Astrophys. 189, 34. 
Barbuy, B., Spite, F., Spite, M., Milone, A. (1990), these proceedings.

Bessell, M.S. (1990), these proceedings.

Bica, E., Alloin, D., Santos, J.F.C. Jr. (1990), Astron. Astrophys. in press.

Bolte, M. (1987), Astrophys. J. 315, 469.

Butler, D., Demarque, P., Smith, H.A. (1982), Astrophys. J. 257, 592.

Cayrel, R. (1986), Astron. Astrophys. 168, 81.

Cohen, J. G. (1982), Astrophys. J. 258, 143.

Da Costa, G. (1990), these proceedings.

de Boer, K.S., Spite, F., Stasinska, G. (eds.) (1989), Recent developments of Magellanic Cloud Research, (Observatoire de Paris: Paris).

Dennefeld, M. (1989), Recent developments of Magellanic Cloud Research, K. de Boer et al. (eds.) (Observatoire de Paris: Paris) p.107.

Dufour, R.J. (1984), IAU Symp. 108 Structure and Evolution of the Magellanic Clouds, S. Van den Bergh, K.S. de Boer (eds.) (Kluwer: Dordrecht) p.353.

Feast, M.W. (1989), The world of galaxies, H.G. Corwin, L. Bottinelli (eds.) (Springer) p.118.

Gratton, R.G., Ortolani, S. (1988), Astron. Astrophys. Suppl. Ser. 73, 137.

Hardy, E., Buonanno, R., Corsi, C.E., Janes, K.A., Schommer, R.A. (1984), Astrophys. J. $278,592$.

Jüttner, A., Reitermann, A., Stahl, O., Wolf, B. (1989), Astron. Astrophys. Suppl. Ser. 81, 93.

Mathis, J.S. (1985), Astrophys. J. 291, 247.

Matteucci, F., Francois, P. (1989), M.N.R.A.S. 239, 885.

Meatheringham, S.J., Dopita, M.A., Ford, H.C., Webster, B.L. (1988), Astrophys. J.327, 651.

Pagel, B., Edmunds, M.G., Fosbury, R.A.E., Webster, B. L. (1978), M.N.R.A.S. 184, 569.

Reitermann, A., Baschek, B., Stahl, O., Wolf, B. (1990), Astron. Astrophys. 234, 109.

Rich, R.M., Da Costa, G.S., Mould, J.R. (1984), Astrophys. J. 286, 517.

Richtler, T., Nelles, B. (1983), Astron. Astrophys. 119, 75.

Richtler, T., Spite, M., Spite, F. (1989), Astron. Astrophys. 225, 351 (paper IV).

Richtler, T., Spite, M., Spite, F. (1990), these proceedings.

Russell, S.C., Bessell, M.S. (1989), Astrophys. J. Suppl. Ser. 70, 865.

Russell, S.C., Dopita, M.A. (1990a), Astrophys. J. Suppl. Ser. 74, 93.

Russell, S.C., Dopita, M.A. (1990b), preprint.

Sagar, R., Pandey, A.K. (1989), Astron. Astrophys. Suppl. Ser. 79, 407.

Schommer, R.A. (1990), these proceedings.

Seggewiss, W., Richtler, T. (1989), Recent developments of Magellanic Cloud Research, K. de Boer et al. (eds.) (Observatoire de Paris: Paris) p.45.

Smith, V.V., Lambert, D.L. (1989), Astrophys. J. 345, L75.

Spite, F., Spite, M., François, P. (1989a), Astron. Astrophys. 210, 25 (paper I)

Spite, M., Spite, F. (1990), these proceedings.

Spite, M., Barbuy, B., Spite, F. (1989b), Astron. Astrophys. 222, 35 (paper III).

Spite, M., Cayrel, R., François, P., Richtler, T., Spite, F. (1989a), Astron. Astrophys. 210, 25 (paper I).

Stryker, L.L., Da Costa, G.S., Mould, J.R. (1985), Astrophys. J. 298, 544.

Westerlund, B.E. (1990), Astron. Astrophys. Rev. 2, 29.

Wolf, B., Jüttner, A., Stahl, O., Baschek, B. (1990), these proceedings. 